\title{
Reconstructing Vegetation Temperature Condition Index Based on the Savitzky-Golay Filter
}

\author{
Manman Li and Junming Liu \\ College of Information and Electrical Engineering, \\ China Agricultural University, \\ Beijing 100083, China \\ liujunming2000@163.com
}

\begin{abstract}
Vegetation temperature condition index (VTCI) is a near-real-time drought monitoring approach which is derived from normalized difference vegetation index (NDVI) changes in a given region to land surface temperature (LST) changes of pixels with a given NDVI value. It can be physically explained as the ratio of temperature differences among the pixels which have the same NDVI values. Due to the noise in the NDVI and LST, results of VTCI have much deviation. The Savitzky-Golay filter, a weighted moving average filter as a polynomial of a certain degree, is applied to smooth out noise in NDVI and LST time-series. VTCI were taken into Guanzhong Plain of Shaanxi Province of each 10-days from March to May in 2007 and 2008 as the study data. In order to reconstruct VTCI space-time (temporal and spatial) series data, the Savitzky-Golay filter was used to reconstruct the VTCI time-series of each pixel in remote sensing images. Then, the results were extended to the surface from the point. The results show that the Savitzky-Golay filter could improve the quality of VTCI and could get a better drought monitoring result.
\end{abstract}

Keywords: Savitzky-Golay filter,VTCI, drought monitoring.

\section{Introduction}

Drought is a long-term, gradual change natural disaster and occurs slowly and complexly. Drought is always induced by water shortage. Drought causes the most yearly economic loss among all natural disasters in China. Thus, drought monitoring plays a very important role in understanding the level and distribution range of drought, which could help in putting forward the measurements against drought in time.

Remote sensing technology could monitor a wild range surface using information of spectroscopy, time, space and direction in objects' surface. On the basis of the multi-spectral characteristics of image, the high spatial resolution and the periodicity of remote sensing information, the research of vegetation and ground coverage become more extensive and more intensive as compared with before. Currently the drought monitoring models are established by vegetation index.

Based on the regional distribution of the scatter diagram of remotely retrieved normalized difference vegetation index (NDVI) and land surface temperature (LST) present like triangular, vegetation temperature condition index (VTCI) is developed for 
monitoring drought occurrence at a regional scale by Wang Pengxin [1]. VTCI is applied in the drought monitoring of Guanzhong Plain of Shaanxi Province and the excellent result has been obtained.

However, residual noise in the NOAA-AVHRR remote sensing image will be remained into the computation of VTCI, though strict pre-processing is applied. The further analysis will impeded and un-correct results might be generated. In this model, the 10-days Maximum Value Composite (MVC) products is used, however, there is much noise in the data sets used in computing VTCI, which may affect the further analysis. Before taking the data to the research, the noise in the data needs to be smoothed out. Lu Linlin used Savitzky-Golay filter to reconstruct NDVI time-series in analyzing the extraction method of winter wheat phonology [2], and it smoothed out noise in the data and loosed less information in the original data.

The phonology information, gotten from NDVI fitted by Savitzky-Golay filter, was more similar with the realistic phonology. The 10-days maximum value composite (MVC) SPOT-VEGETATION dataset was used as main remotely sensed data from 1999 to 2005 in Gu's research [3]. The Savizky-Golay filter, which could improve the representation of the growth curve of winter wheat growth by NDVI time-series curve, was used to eliminate the noise. Gong Pan used the Savizky-Golay filter to smooth the 10-days compositing data and the results indicate that the time-series data has better correlational relationship with the vegetation growth [4].

This study was intended to introduce the Savizky-Golay filter into VTCI data. The objectives were: To smooth out noise before computing VTCI and to reconstruct NDVI and LST time-series with Savitzky-Golay filter. Thus, more accurate results of drought monitoring could be obtained.

\section{Methodology}

\subsection{Study Area and Data}

For this study, proper research region is needed. The region with large range of vegetation coverage and soil surface water content from wilting water content to field capacity are needed for drought monitoring with VTCI. Thus, Guanzhong Plain is selected for the study (Fig.1). Guanzhong Plain is located in the center of Shaanxi province and between Qinling Mountains and Shanbei Plateau. Guanzhong Plain is a graben structural plain, flat and fertile, and is one of the most important wheat and cotton growth areas.

NOAA-AVHRR images day transited from March to May in 2007 and 2008 were choosed. Firstly, geometric correction was done in two steps: (1) revising the distortion of pixels; (2) using a traditional geometric correction method - polynomial correction method. The size of the pixel of AVHRR image after resembling with bilinear interpolation is $1.1 \mathrm{~km} \times 1.1 \mathrm{~km}$. The projection method is Lambert equal area projection. By using AVHRR data through atmospheric correction, radiometric correction and calibration, we can transform data of band 1 and band 2 into the surface reflectivity and data of band 3, 4 and 5 into the surface radiance. Then we can calculate the brightness temperature of band 3,4 and 5 by center wavelength technology. Lastly, NDVI and LST can be calculated severally. 


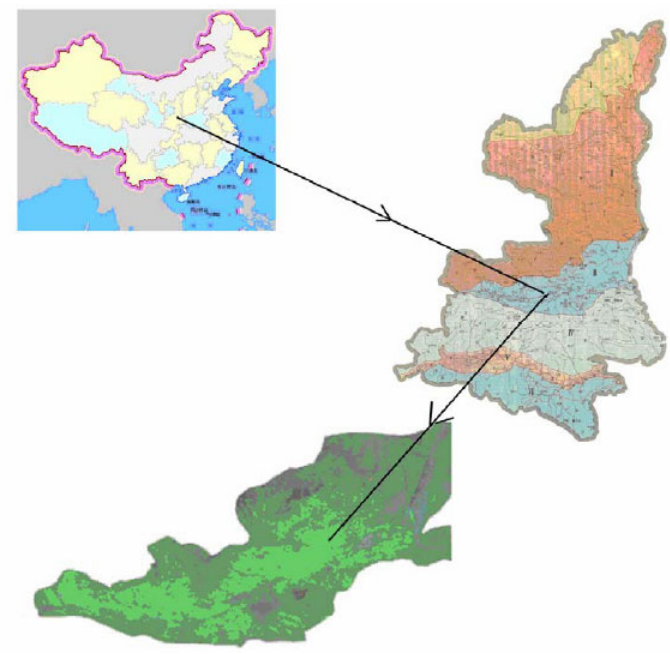

Fig. 1. The study area

\subsection{Vegetation Temperature Condition Index}

Wang Pengxin proposed the concept of vegetation temperature condition index (VTCI) for monitoring drought [5]. The index can be used to monitor drought occurrence at a regional level for a special period (e.g. 10 days) of a year, and to capture spatial variatious of drought occurrence within the region. The new drought monitoring index is not only related to NDVI changes in a region, but also give emphasis on land surface temperature changes of pixels which have the same NDVI value. The index can be defined as [1]:

$$
V T C I=\frac{L S T_{N D V I_{i} \cdot \max }-L S T_{N D V I_{i}}}{L S T_{N D V I_{i} \cdot \max }-L S T_{N D V I_{i} \cdot \min }}
$$

There into,

$$
\begin{aligned}
& L S T_{N D V I_{i} \cdot \max }=a+b \cdot N D V I_{i} \\
& L S T_{N D V I_{i} \cdot \min }=a^{\prime}+b^{\prime} \cdot N D V I_{i}
\end{aligned}
$$

In the formula, $L S T_{N D V_{i} \text { max }}, L S T_{N D V \text { min }}$ are maximum and minimum of land surface temperature when $\mathrm{NDVI}_{\mathrm{i}}$ is equal to a particular value; $\mathrm{LST}_{\mathrm{NDVIi}}$ is land surface temperature of a pixel when the value of NDVI is NDVI $;$; a, b, a', b' are undetermined coefficients. In this research, we can get the coefficients approximately in formula (2) in the scatter diagram of NDVI and LST of the study area.

The value of VTCI is from 0 to 1.The lower value of VTCI, the heavier the drought occurrence is. The index is site-specific and time-specific. 


\subsection{The Savitzky-Golay Filter}

The Savitzky-Golay filter, called SG filter for short, is a simplified least-squares-fit convolution for smoothing and computing derivatives of a set of consecutive values, proposed by Savitzky and Golay in 1964 [8]. It can be understood as a weighted moving average filter with weighting given as a polynomial of a certain degree. This polynomial is designed to preserve higher moments within the data and to reduce the bias introduced by the filter. This filter can be applied to any consecutive data when the points of the data are at a fixed and uniform interval along the chosen abscissa, specially the time-series data. The general equation of the simplified least-squares convolution for time-series data smoothing can be given as follows[9]:

$$
Y_{i} *=\sum_{j=-m}^{j=m} C_{j} Y_{j+i}
$$

where $\mathrm{Y}$ is the original time-series data value, $\mathrm{Y} *$ is the resultant time-series data value, $\mathrm{C}_{\mathrm{i}}$ is the coefficient for the ith time-series data value of the filter (smoothing window).The index $\mathrm{i}$ is the running index of the original ordinate data table. The smoothing array (filter size) consists of $2 m+1$ points, where $m$ is the half-width of the smoothing window.

There are two parameters that must be determined according to the time-series data observations, when the filter is applied. The first parameter is $\mathrm{m}$, the half-width of the smoothing window. Usually, a larger value of $\mathrm{m}$ produces a smoother result at the expense of flattening sharp peaks. The second parameter is $d$, the degree of the smoothing polynomial. A smaller value of $d$ will produce a smoother result but may introduce bias. And a higher value of $d$ will reduce the filter bias, but may over fit the data and give a noisier result.

\section{Experimental Results}

\subsection{Reconstruct Time-Series}

Select a pixel in $\mathrm{Xi}^{\prime}$ an, whose geographic coordinate is $\left(108^{\circ} 56^{\prime} \mathrm{E}, 34^{\circ} 18^{\prime} \mathrm{N}\right)$. The parameters are set to $(2,3)$ and $(3,4)$. Fig. 2 shows the change of the NDVI and LST time-series fitted by the Savitzky-Golay filter with different parameters in contrast. Obviously, the flitted time-series could pass through the original time-series and remove the low value in the original time-series. A certain extent, the Savitzky-Golay filter plays a role in eliminating noise.

From the comparison, it shows that the results of two different parameters are very close, the difference is not significant. The result got from parameters $(2,3)$ is little larger than the result got from parameters $(3,4)$. However, noise usually depress time-series value. The top value may keep more realistic information. Sun Wei et al. monitored drought of Guanzhong Plain in each early ten days of May in recent five years and the result show that VTCI is close to the amount of precipitation in recent 20 


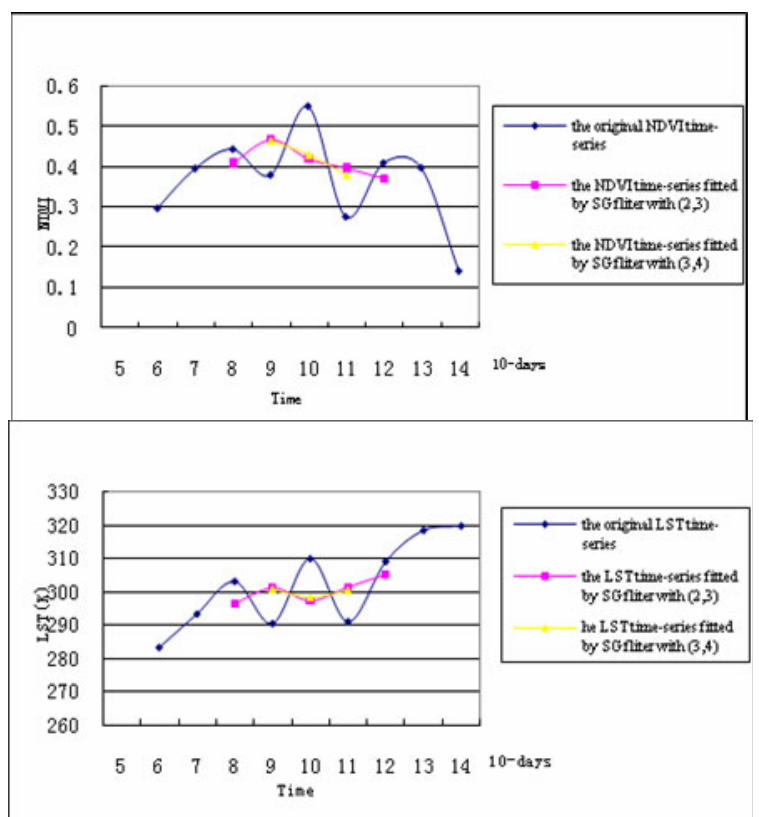

a. Comparison of time-series before and after fitting by the Savitzky-Golay filter with different parameters from March to May in 2007

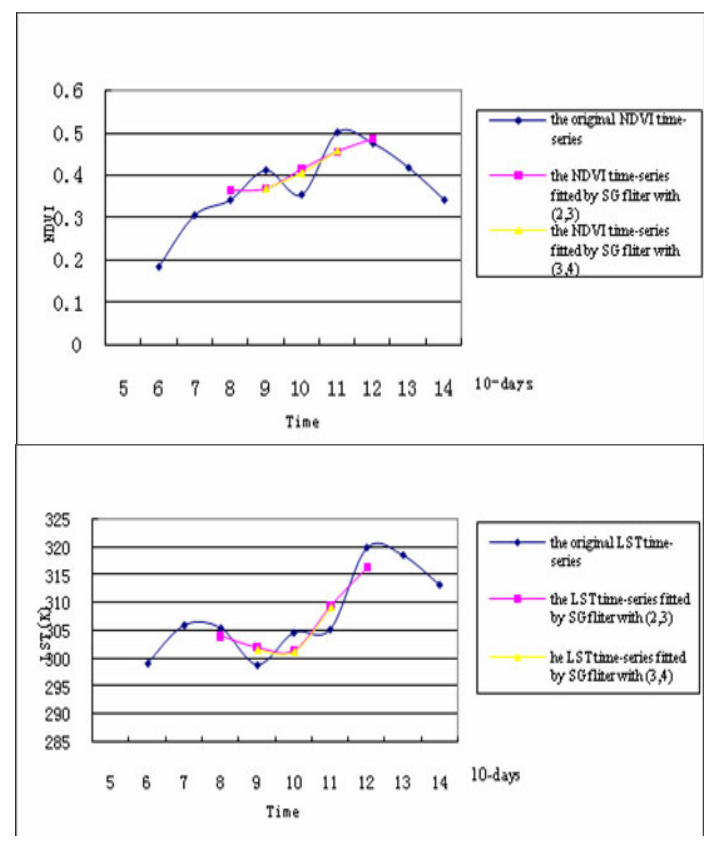

b. Comparison of time-series before and after fitting by the Savitzky-Golay filter with different parameters from March to May in 2008

Fig. 2. The change of time-series before and after fitting by the Savitzky-Golay filter with different parameters 
days[6], indicating that the VTCI is a near-real time drought monitoring approach. When there is little difference in the results of two parameters, when $m$ is set as 2, more data could be gotten. Considering that, when fit the NDVI and LST time-series by the Savitzky-Golay filter, the parameters set as $(2,3)$ in the study.

\subsection{VTCI Image}

Based on formula (1), when VTCI is computed with NDVI and LST is fitted by the Savitzky-Golay filter, images were generated. Comparative results are in fig. 3 and fig. 4.
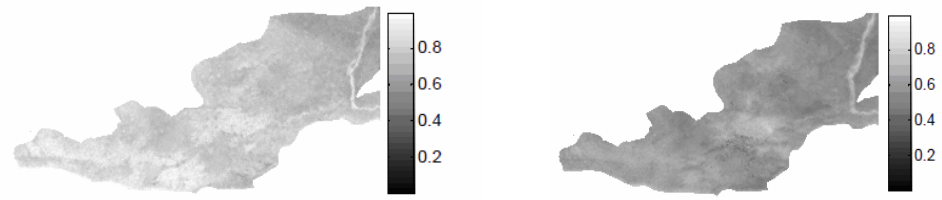

a. the original VTCI image and the fitted image in the early ten days of April,2007
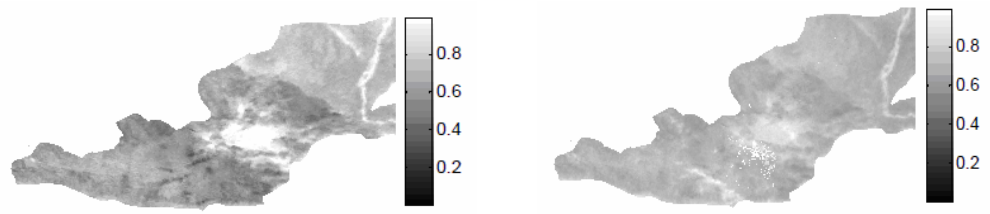

b. the original VTCI image and the fitted image in the middle ten days of April,2007
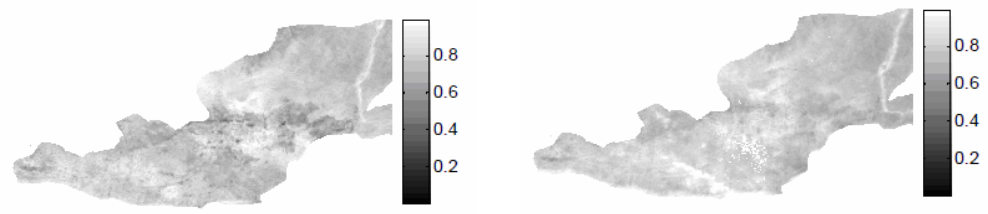

c. the original VTCI image and the fitted image in the early ten days of May,2007

Fig. 3. Comparison of VTCI images in 2007 on the base of the agricultural weather data of Shaanxi Meteorology

By the data from the agricultural meteorological station of Shaanxi Meteorology Bureau, from the last ten days of March to the middle ten days of May in 2007, most areas of Shaanxi province showed higher temperatures. Compared with the average year, the amount of precipitation decreased about $20 \%$ to $70 \%$. The entropy in soil loosed fast and the development of drought is continuous. The drought area of Guanzhong Plain extended from east to west gradually. The study area had precipitation in the early ten days of April, though it was not enough for releasing drought, it helped in increasing the water content of the soil surface. Fig. 3 shows that VTCI images fitted by the Savitzky-Golay filter could get a better monitoring result. 

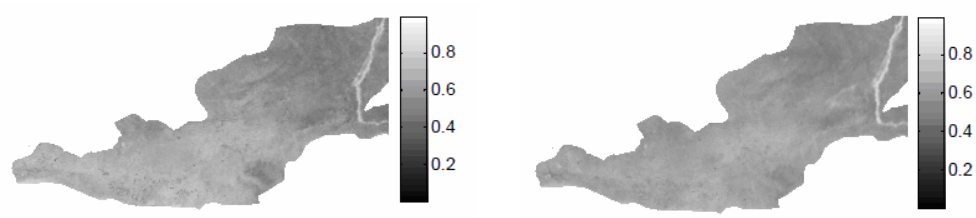

a. the original VTCI image and the fitted image in the early ten days of April,2008
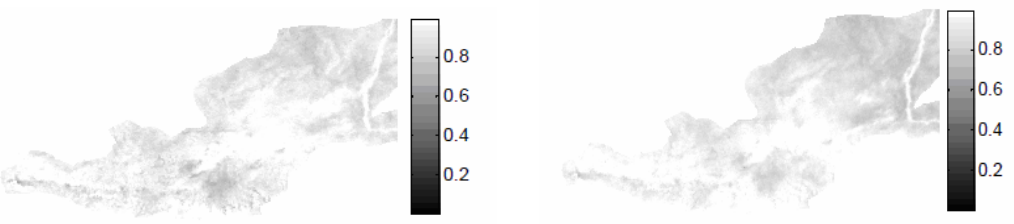

b. the original VTCI image and the fitted image in the middle ten days of April,2008
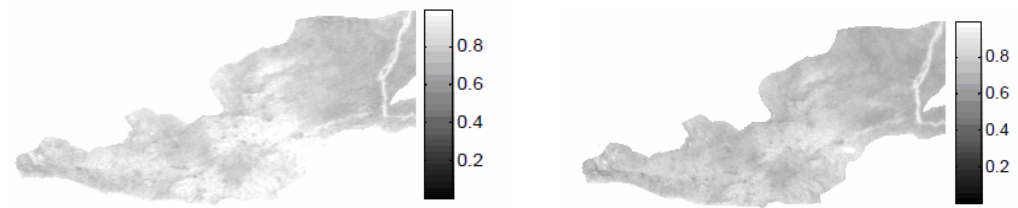

c. the original VTCI image and the fitted image in the last ten days of April,2008

Fig. 4. Comparison of VTCI images in 2008

From March to May in 2008, the average temperature of Guanzhong Plain was a little higher than average year. The total rainfall amount of the midwest of Guanzhong Plain was 50--100mm, less than average year[12]. Guanzhong Plain has irrigation conditions that could help in releasing drought. VTCI images fitted by the Savitzky-Golay filter could get a better monitoring result (fig. 4). There was a larger process of precipitation in the middle of April, which increased the water content of the land. So, the value of VTCI of monitoring results increased.

Guanzhong Pain has higher probability of spring drought and summer drought. And the drought in the east is worse than in the west. Based on this trend, the VTCI images fitted by Savitzky-Golay filter could reflect this better.

The noise usually depresses the value. The Savitzky-Golay filter could smooth out the noise more or less and remove the low value. The VTCI images fitted by the Savitzky-Golay filter are more close to the truth.

\section{Conclusion}

The Savitzky-Golay filter is a simplified least-squares-fit convolution and a weighted moving average filter. The time-series data fitted by the Savitzky-Golay filter can smooth out noise in time-series, specifically that caused primarily by cloud contamination and atmospheric variability. The experiment with NOAA images from March to May in 2007 and 2008, indicate that the VTCI calculated with the NDVI and the LST time-series fitted by the Savitzky-Golay filter, can improve the quality of VTCI and get a better drought monitoring result. 
Selection of parameters is extremely important when use the Savitzky-Golay filter to reconstruct NDVI time-series. That affects the accuracy of the results directly. Selection of parameters in this study was determined by the study data and taken into the actual situation on the ground. Subject to the limitations of the data, we just use the Savitzky-Golay filter simply in the study. If there are the whole year VTCI data, higher accuracy VTCI data could be gotten in the fitting iteration process with the Savitzky-Golay filter.

VTCI is a real-time approach, and has a certain correlation with the soil water content. When there is a precipitation, even it's not enough to release the drought completely, but it increases the soil water content. The results obtained through remote sensing monitoring show no drought happen. That affects the accuracy of the results. It's worth to study how to reduce the influence. At the same time, it's a research direction that fills the missing data within the region through the Savitzky-Golay filter.

Acknowledgments. This research was supported by the National Natural Science Foundation of China (Grant No. 40871159 and 40571111), China National High Technology Research and Development Program (Grant No. 2007AA12Z139), and the European Commission (Call FP7-ENV-2007-1 212921) as part of the CEO-AEGIS project.

\section{References}

1. Wang, P., Gong, J., Li, X.: Vegetation-Temperature Condition Index and Its Application for Drought Monitoring. Geomatics and Information Science of Wuhan University, 412-419 (2001)

2. Lu, L., Guo, H.: Extraction method of winter wheat phenology from time series of SPOT/VEGETATION data. Transactions of CASE, 174-181 (2009)

3. Gu, X., Song, G., Han, L., Xu, C., Pan, Y.: Monitoring of growth changes of winter wheat based on change vector analysis. Transactions of the CSAE, 159-167 (2008)

4. Gong, P., Tang, H., Chen, Z., Zhang, F.-r.: Land Cover Classification based on MODIS NDVI \& LST Time Series Data in Northeast China. Resources Science, 204-211 (2006)

5. Wang, P., Sun, W.: Researches and Applications on Drought Monitoring Approach of Vegetation Temperature Condition Index. Science \& Technology Review, 56-59 (2006)

6. Sun, W., Wang, P., Han, L., Yan, K., Zhang, S., Li, X.: Further improvement of the approach to monitoring drought using vegetation and temperature condition indexes from multi-years remotely sensed data. Transactions of the CSAE, 22-24 (2006)

7. Sun, W.: Quantification of the vegetation temperature condition index drought monitoring approach. China Agricultural University (2008)

8. Savitzky, A., Golay, M.J.E.: Smoothing and differentiation of data by simplified least squares procedures. Analytical Chemistry, 1627-1639 (1964)

9. Press, W.H., Teukolsky, S.A., Vetterling, W.T., Flannery, B.P.: Numerical Recipes in C The Art of Scientific Computing, 2nd edn., pp. 650-655. Cambridge University Press, Cambridge (2002)

10. Fan, R., Chen, Y., Wei, J.: An Analysis of Drought Features in Shaanxi Provine. Journal of xi'an University of Technology, 200-207 (1996) 
11. Zhang, H., Chen, H., Zhou, G., Zhao, B., Gao, Z.: Application of Normalized Multi-band Drought Index Method in Cropland Drought Monitoring. Science \& Technology Review, 23-27 (2009)

12. Cai, X., Lei, X., Tian, W., Mao, M.: Evaluation of Climate Influence in Shaanxi Provine in 2008. Shaanxi Weather, 21-25 (2009)

13. Madden, H.: Comments on the Savitzky -Golay convolution method for least-squares fit smoothing and differentiation of digital data. Analytical Chemistry, 1383-1386 